\title{
Natural Compound Formula RM88 Significantly Reduces Blood Alcohol Concentration in Humans
}

\author{
E. Russell Vickers \\ Clinical Stem Cells PL, Sydney, Australia \\ Email: manager@clinicalstemcells.com
}

How to cite this paper: Vickers, E.R. (2020) Natural Compound Formula RM88 Significantly Reduces Blood Alcohol Concentration in Humans. Pharmacology \& Pharmacy, 11, 105-117.

https://doi.org/10.4236/pp.2020.116010

Received: May 31, 2019

Accepted: June 26, 2020

Published: June 29, 2020

Copyright $\odot 2020$ by author(s) and Scientific Research Publishing Inc. This work is licensed under the Creative Commons Attribution International License (CC BY 4.0).

http://creativecommons.org/licenses/by/4.0/ (c) (i) Open Access

\begin{abstract}
There is extensive worldwide use for the social consumption of alcohol. Abuse of alcohol causes substantial personal, psychological and medical health issues. In addition, there are significant national economic costs from lost productivity. However, there are limited pharmaceutical drugs for the treatment of alcohol overuse. Historically, many cultures have used herbs and other natural compounds to reduce problematic alcohol induced behaviour but the evidence is anecdotal. This study investigated if a natural compound formula (RM88) that was developed could reduce blood alcohol concentration (BAC) in a controlled case series. Thirteen subjects ( 5 males, 8 females, age range 18 to 85 years) completed 16 paired sessions of alcohol only versus RM88 with alcohol. Subjects consumed one to three standard drinks of beer, wine or spirits ( 14.7 to $29.4 \mathrm{gm}$ alcohol). Measurements were made by a fuel cell breathalyzer for a period of 90 minutes. Summated BAC showed a reduction in $94 \%(15 / 16)$ of paired test sessions (BAC reduction range $23 \%-79 \%$, mean $50.9 \% \pm 16.5 \%, \mathrm{p}=0.0005$ ). Data normalized to $20 \mathrm{gm}$ alcohol (two standard drinks) showed a significance of $p=0.00026$. One subject on prednisone and hydroxychloroquine drugs had increased BAC from RM88. The average reductions of BAC for the beverages were spirits $34 \%(n=3)$, beer $36 \%(\mathrm{n}=3)$, and wine $52 \%(\mathrm{n}=10)$. RM88 showed that this combination of natural compounds was very effective in reducing maximal peak concentrations of alcohol.
\end{abstract}

\section{Keywords}

Alcohol, Blood Alcohol Concentration, Herbal Medicine, Polyphenols, Natural Compounds 


\section{Introduction}

Alcohol (ethanol, ethyl alcohol) in its social context of use is widely consumed in many countries in the form of spirits, beer and wine. Current data shows the top 10 countries consuming 10.6 to 14.4 litres of pure alcohol per person each year [1]. The purpose of drinking alcohol is mainly associated with social interaction and celebration. However, abuse of the substance can lead to significant issues of alcoholism, and physical and psychological deterioration [2]. In addition, the economic cost of lost productivity from alcohol is considerable and in a systematic review the burden of alcohol is estimated to be $0.45 \%$ to $5.44 \%$ of Gross Domestic Product (GDP, $\mathrm{n}=12$ countries) [3]. Methods to attenuate alcohol toxicity have been recently described [4]. First-line pharmaceutical drugs to treat chronic alcoholism are naltrexone and acamprosate, with disulfiram as a second-line "punishment" drug due to issues of compliance and toxicity and where "hangover" effects are deliberately magnified to prevent recurrence [5].

From a medical perspective, the chronic use of alcohol has been causative or associated with 3 million deaths each year and over 200 medical conditions [6]. Recent systematic reviews have also detailed the wide-ranging damaging effects of chronic alcohol exposure in regenerative medicine by inhibitory mechanisms on stem cell function [7] [8]. Other public health issues include alcohol causation for fetal alcohol spectrum disorders, and cancer attributed to the toxic ethanol metabolite acetaldehyde [9]. Alcohol consumption has been an integral part of human interactions for thousands of years, with evidence that alcohol was consumed in China 10,000 years ago. Social meetings have important psychological benefits and can be associated with a moderate intake of alcohol. Many countries rely on taxes obtained from alcohol, and realistically, alcohol consumption will continue indefinitely for a high percentage of the world population.

Blood alcohol concentration (BAC) measurement by a breathalyzer device is a convenient, portable and validated method used by law enforcement to establish the concentration where cognitive effects are detrimental to driving a motor vehicle, powered marine craft, and aircraft. BAC is also used to assess unacceptable social behaviour and exclude work personnel affected by alcohol from office, manufacturing and construction sites. There has been no proven scientific method to defeat or "cheat" a breathalyzer device except for brief hyperventilation. The breathalyzer affords rigor and quantitative repeated measurements for human experimental use and has been applied extensively in methodology in the scientific literature. The pharmacokinetics and metabolic pathways of alcohol have been reported extensively. The principal pathway following absorption of ethanol from stomach and small intestine is a first-order kinetic process and subsequent liver metabolism by alcohol dehydrogenase (ADH) to yield acetaldehyde, then further enzymic cleavage by acetaldehyde dehydrogenase (ALDH) to yield acetate and water [10].

Herbal medicines and other natural compounds such as vitamins and miner- 
als have proven popular with the general public to maintain good health. Evidence ranges from well controlled studies to empirical evidence. Several studies have investigated herbs for their effect on BAC. An early study that investigated the effect of the Asian herb Panax Ginseng (PG) found improved clearance of blood alcohol in $71 \%$ of subjects on a concurrent intake of $3 \mathrm{gm}$ PG with alcohol and suggested clearance via the secondary cytochrome P-450 pathway [11]. A later study using a similar dose of 3.2 gm PG showed a 20\% BAC reduction $(0.05 \%$ to $0.04 \% \mathrm{BAC})$ at 30 minutes after alcohol/PG consumption [12]. PG however has numerous and frequent side effects from high dose and chronic use and include morning diarrhea (35\%), skin eruption (25\%), nervousness (25\%), sleeplessness (20\%), and hypertension (17\%) ( $\mathrm{n}=133$ patients) [13] [14]. Other animal studies of the Chinese herb NPI-028 (using high dose $1.5 \mathrm{~g} / \mathrm{kg}$, rats) reduced alcohol cravings with similar reports of the polyphenols of daidzin [15] and the psychedelic ibogaine [16].

In summary there has been minimal evidence-based research on herbal and natural compounds to reduce alcoholism in humans. Many countries have anecdotal "folklore" evidence of their indigenous cultures using traditional herbal remedies in an attempt to prevent intoxication [4]. The purpose of this study was to evaluate if a combination of herbal powders, polyphenols and other natural products could be effective in reducing BAC as measured by a breathalyzer. The scope of the preliminary investigation for compounding the formula covered the extensive domain of herbs and polyphenols (>20,000 known compounds), peptides $(>50,000)$, fungi, vitamins, minerals and amino acids. Specifically, the developed formula in this study was a new formula and did not include the previously reported Panax Ginseng, Chinese herb NPI-028, daidzin or ibogaine.

\section{Materials and Methods}

\subsection{Subjects}

The study recruited 13 consenting adult subjects, 8 females and 5 males, age range 18 to 85 years (Table 1). Subjects were in good general health and reported a history for the social intake of alcohol of one to five standard drinks per

Table 1. Characteristics of subjects.

\begin{tabular}{cccccccccccccc}
\hline Subject & 1 & 2 & 3 & 4 & 5 & 6 & 7 & 8 & 9 & 10 & 11 & 12 & 13 \\
\hline Gender & M & M & M & F & F & F & F & M & M & F & F & F & F \\
Age (years) & 65 & 60 & 63 & 51 & 29 & 58 & 18 & 29 & 63 & 60 & 46 & 85 & 50 \\
Weight (kg) & 73 & 84 & 78 & 57 & 62 & 56 & 48 & 85 & 70 & 87 & 74 & 65 & 80 \\
Height (cm) & 177 & 175 & 180 & 160 & 156 & 165 & 154 & 182 & 178 & 165 & 163 & 150 & 165 \\
Medication & & $\mathrm{M}$ & & $\mathrm{P}, \mathrm{H}$ & & Pro & & & $\mathrm{R}, \mathrm{F}$ & $\mathrm{L}, \mathrm{R}, \mathrm{F}$ & & & \\
\hline
\end{tabular}

Medication legend: $\mathrm{M}$ metformin, $\mathrm{P}$ prednisone, $\mathrm{H}$ hydroxychloroquine, Pro propanolol, $\mathrm{R}$ ramipril, $\mathrm{F}$ felodipine, L lisdexamfetamine. 
week. No subject had a history of alcohol abuse. Several subjects were taking prescription medication for medical conditions that were well controlled. There were no medical contraindications or known significant medication interactions with alcohol in the cohort. The trial design was an open label paired intrasubject comparison study measuring BAC over two separate sessions.

The first session had the subject drink only alcohol (control), the second session had the subject consume a natural compounded formula and alcohol (active). Sessions were at least two days apart. The study was conducted during 2020 in Australia with appropriate study measures in place due to coronavirus.

\subsection{Herbal Test Product}

Herbal and natural compounds were selected that could potentially reduce alcohol, improve liver health and enhance regenerative medicine procedures. All compounds were non-prescription (Australian regulations) and were available in the form of powders that were purchased from Australian licensed health food shops, supermarkets and pharmaceutical retail outlets, and overseas suppliers. The combination formula was named RM88 (Regenerative Medicine trial code number 88). The herbal powder combination was added to a standard cookie recipe of coconut flour, egg, stevia sweetener, chocolate buds and peanut butter. The cookie was eaten 60 minutes before alcohol consumption to allow for absorption of the ingredients. Most sessions used RM88 in the cookie form, and several sessions used the RM88 mixed in water as a drink.

\subsection{Alcohol Intake}

Liquor was in the form of wine, beer or premium spirits (whisky or gin) and purchased from licensed liquor supply shops. Liquor in the quantity range of one to three standard drinks was consumed where a standard drink contains 10 gm alcohol. Subjects were instructed to drink the liquor within 20 minutes. To replicate a social environment subjects if desired could consume water and small amounts of food during alcohol consumption and BAC data collection. Time taken to consume the alcohol and any food intake was recorded in the control session and duplicated in the active RM88 sessions. The BAC was recorded at multiple time points after the completion of drinking, every 5 minutes for 1 hour, followed by every 10 minutes for a further 30 minutes. Several sessions were extended until BAC reached $0 \%$. At the end of alcohol consumption subjects rinsed and swallowed their mouth with water to reduce any residual oral mucosal alcohol. BAC was measured by a high quality fuel cell breathalyzer unit (LifeGuard, Model CA1 645009, USA distributed by breathalyser.com.au and with approved Australian Standard code AS 3547). Alcohol intake was calculated in $\mathrm{gm}(10 \mathrm{ml}$ alcohol $=8 \mathrm{gm})$.

\subsection{Statistics}

Chi square and paired $t$ test were used with a level of significance set at $\mathrm{p}<0.05$. 


\section{Results}

There were 16 paired sessions completed by the 13 subjects with an intake range 14.7 to $29.4 \mathrm{gm}$ alcohol. Alcohol consumed was in the form of wine ( $\mathrm{n}=10$ paired sessions), beer $(n=3)$, and premium spirits $(n=3)$ (Table 2). The group was considered as a representative sample of the population, with mixed males and females over a wide age range. Results of group analysis demonstrated that RM88 had a significant effect on reducing BAC on several measures. Totals of paired BAC measurements were summated and showed a reduction in alcohol over the measured time period in $94 \%(15 / 16)$ of paired sessions from $23 \%$ to $79 \%$ (mean $50.9 \% \pm 16.5$ ) (chi square $\mathrm{p}=0.0005$ ), with one subject (on prednisone and hydroxychloroquine drugs) increasing the alcohol concentration from the active formula by 39\%. Results analyzing paired sessions (where $t$ test $\mathrm{p}<$ $0.05)$ showed that RM88 was effective in $81 \%$ (13/16) of drinking sessions to reduce BAC concentration (chi square $\mathrm{p}=0.012$ ). Table 3 shows data normalized to $20 \mathrm{gm}$ alcohol for control versus RM88 sessions ( $\mathrm{p}=0.00026)$, and where one standard drink = $10 \mathrm{gm}$ alcohol (Figure 1). Three additional sessions were recorded later in the study where the RM88 was consumed 20 minutes before drinking alcohol and the three sessions showed improved alcohol reduction compared with the cookie consumed 60 minutes before alcohol ( $\mathrm{p}<0.05)$. The total BAC changes from RM88 were similar between males and females $(45.9 \% \pm$ $18.7 \% ; 45.6 \% \pm 24.7 \%$ ), respectively. There was no correlation between age and $\mathrm{BAC}$ reduction from $\mathrm{RM} 88$ (Pearson's $\mathrm{R}=-0.034$ ). The average reduction of BAC for the beverages were for spirits $34 \%(n=3)$, beer $36 \%(n=3)$, and wine $52 \%(n=10)$.

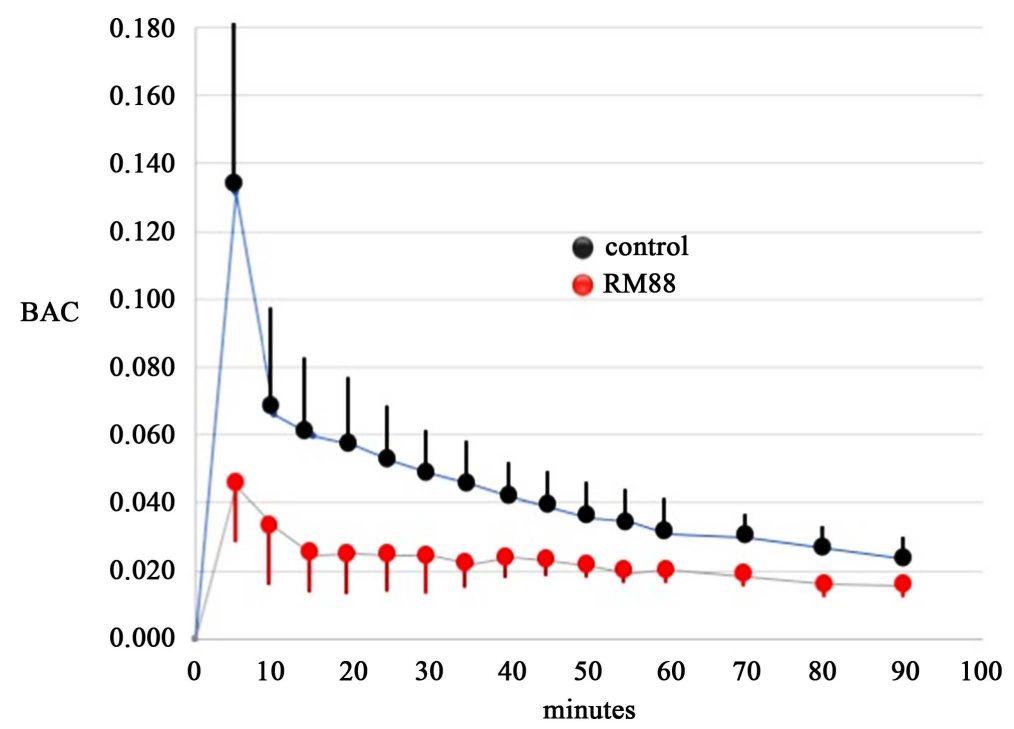

Figure 1. Normalized blood alcohol concentration (BAC) data of Control and RM88 sessions expressed as means (SD). Data normalized to 20 gm alcohol per session and measured for 5 - 90 minutes $(\mathrm{p}=0.00026)$. One standard drink $=10 \mathrm{gm}$ alcohol. Actual mean of consumed alcohol in 16 sessions was $22.1 \pm 2.7 \mathrm{gm}$ alcohol. At the 5 minute Control data point the \pm standard deviation bar $(0.093)$ was truncated. 
Table 2. Blood alcohol concentration (BAC) for sessions 1 - 16 control (C) versus active (A) RM88. Corresponding missing values were paired and not included in statistical analyses. Several subjects were able to complete both sessions to $0 \%$ BAC. Subject 2 undertook paired sessions $2-5$, all other subjects only one paired session. Session 7C/7A showed an increase in BAC by $+39 \%{ }^{*}$.

\begin{tabular}{|c|c|c|c|c|c|c|c|c|c|c|c|c|c|c|c|c|}
\hline Session & $1 \mathrm{C}$ & $1 \mathrm{~A}$ & $2 \mathrm{C}$ & $2 \mathrm{~A}$ & $3 C$ & $3 \mathrm{~A}$ & $4 \mathrm{C}$ & $4 \mathrm{~A}$ & $5 \mathrm{C}$ & $5 \mathrm{~A}$ & $6 C$ & $6 \mathrm{~A}$ & $7 \mathrm{C}$ & 7A & $8 C$ & $8 \mathrm{~A}$ \\
\hline Subject & 1 & 1 & 2 & 2 & 2 & 2 & 2 & 2 & 2 & 2 & 3 & 3 & 4 & 4 & 5 & 5 \\
\hline Alcohol & spirits & spirits & spirits & spirits & wine & wine & beer & beer & beer & beer & beer & beer & spirits & spirits & wine & wine \\
\hline $\mathrm{gm}$ & 19.2 & 19.2 & 19.2 & 19.2 & 28 & 28 & 14.7 & 14.7 & 29.4 & 29.4 & 26.4 & 26.4 & 19.2 & 19.2 & 24 & 24 \\
\hline $5 \mathrm{~min}$ & 0.223 & 0.029 & 0.265 & 0.068 & 0.191 & 0.11 & 0.062 & 0.063 & 0.111 & 0.062 & 0.039 & 0.021 & 0.048 & 0.042 & 0.4 & 0.034 \\
\hline 10 & 0.083 & 0.016 & 0.202 & 0.029 & 0.072 & 0.076 & 0.039 & 0.016 & 0.074 & 0.042 & 0.042 & 0.022 & 0.035 & 0.147 & 0.055 & 0.032 \\
\hline 15 & 0.043 & 0.013 & 0.17 & 0.031 & 0.056 & 0.046 & 0.035 & 0.011 & 0.045 & 0.035 & 0.045 & 0.02 & 0.038 & 0.049 & 0.057 & 0.034 \\
\hline 20 & 0.035 & 0.013 & 0.137 & 0.028 & 0.053 & 0.053 & 0.029 & 0.011 & 0.049 & 0.035 & 0.043 & 0.024 & 0.036 & 0.038 & 0.055 & 0.032 \\
\hline 30 & 0.032 & 0.011 & 0.097 & 0.027 & 0.048 & 0.056 & 0.021 & 0.011 & 0.048 & 0.027 & 0.044 & 0.029 & 0.036 & 0.037 & 0.048 & 0.029 \\
\hline 35 & 0.028 & 0.012 & 0.081 & 0.022 & 0.044 & 0.035 & 0.019 & 0.009 & 0.044 & 0.024 & 0.042 & 0.03 & 0.036 & & 0.047 & 0.028 \\
\hline 40 & 0.028 & 0.018 & 0.071 & 0.023 & 0.045 & 0.033 & 0.015 & 0.01 & 0.041 & & 0.04 & 0.031 & 0.032 & 0.036 & 0.044 & 0.028 \\
\hline 45 & 0.024 & 0.02 & 0.064 & 0.019 & 0.038 & 0.032 & 0.012 & 0.009 & 0.039 & 0.023 & 0.04 & 0.033 & 0.031 & & 0.042 & 0.027 \\
\hline 50 & 0.022 & 0.015 & 0.059 & 0.017 & 0.039 & 0.029 & 0.011 & 0.007 & 0.035 & 0.023 & 0.034 & 0.025 & 0.029 & 0.03 & 0.043 & 0.023 \\
\hline 55 & 0.026 & 0.017 & 0.052 & 0.016 & & & 0.006 & 0.006 & 0.032 & & 0.037 & 0.021 & 0.027 & & 0.038 & 0.022 \\
\hline 60 & 0.026 & 0.015 & 0.047 & 0.014 & 0.032 & 0.024 & 0 & 0.006 & 0.03 & 0.022 & 0.034 & 0.022 & 0.027 & 0.027 & 0.037 & 0.02 \\
\hline 70 & 0.022 & 0.013 & 0.041 & 0.009 & 0.029 & 0.022 & & 0.005 & 0.031 & 0.02 & 0.027 & 0.018 & 0.021 & 0.028 & 0.035 & 0.017 \\
\hline 90 & 0.012 & 0.008 & 0.023 & 0.007 & 0.022 & 0.016 & & & 0.021 & 0.023 & 0.023 & 0.014 & 0.019 & 0.021 & 0.027 & 0.013 \\
\hline 100 & 0.01 & 0.005 & 0.021 & 0.006 & 0.021 & 0.015 & & & 0.02 & 0.019 & 0.019 & 0.013 & 0.015 & 0.017 & 0.025 & \\
\hline 110 & 0.007 & 0.005 & 0.018 & 0.005 & 0.019 & & & & 0.016 & 0.015 & 0.018 & 0.01 & 0.011 & & 0.023 & \\
\hline 120 & 0.006 & 0.005 & 0.016 & 0 & 0.016 & & & & 0.013 & 0.014 & 0.015 & 0.009 & 0.008 & & 0.02 & \\
\hline 130 & 0.006 & 0 & & & 0.764 & & & & 0.012 & 0.011 & 0.013 & 0.007 & 0.006 & & & \\
\hline 140 & & & & & & & & & 0.009 & 0.009 & 0.011 & 0.005 & 0.005 & & & \\
\hline 150 & & & & & & & & & 0.008 & 0.008 & 0.008 & 0 & 0 & & & \\
\hline 160 & & & & & & & & & 0.006 & 0.005 & 0.007 & & & & & \\
\hline 170 & & & & & & & & & 0.005 & 0.005 & 0.006 & & & & & \\
\hline 180 & & & & & & & & & 0 & 0 & 0 & & & & & \\
\hline $\begin{array}{c}\% \text { change } \\
B A C\end{array}$ & & $66 \%$ & & $76 \%$ & & $61 \%$ & & $36 \%$ & & $32 \%$ & & $39 \%$ & & $+39 \%^{*}$ & & $65 \%$ \\
\hline $\begin{array}{c}p \text { value } \\
(t \text { test })\end{array}$ & & 0.033 & & 0.0002 & & 0.070 & & 0.010 & & 0.002 & & $<0.001$ & & 0.245 & & 0.094 \\
\hline $\begin{array}{c}\text { Pearson's } \\
R\end{array}$ & & 0.737 & & 0.931 & & 0.923 & & 0.830 & & 0.973 & & 0.899 & & 0.358 & & 0.425 \\
\hline
\end{tabular}




\section{Continued}

\begin{tabular}{|c|c|c|c|c|c|c|c|c|c|c|c|c|c|c|c|c|}
\hline Session & $9 \mathrm{C}$ & $9 A$ & $10 \mathrm{C}$ & $10 \mathrm{~A}$ & $11 \mathrm{C}$ & $11 \mathrm{~A}$ & $12 \mathrm{C}$ & $12 \mathrm{~A}$ & $13 C$ & $13 \mathrm{~A}$ & $14 \mathrm{C}$ & $14 \mathrm{~A}$ & $15 \mathrm{C}$ & $15 \mathrm{~A}$ & $16 \mathrm{C}$ & $16 \mathrm{~A}$ \\
\hline Subject & 6 & 6 & 7 & 7 & 8 & 8 & 9 & 9 & 10 & 10 & 11 & 11 & 12 & 12 & 13 & 13 \\
\hline Alcohol & wine & wine & wine & wine & wine & wine & wine & wine & wine & wine & wine & wine & wine & wine & wine & wine \\
\hline gm & 24.0 & 24.0 & 20.8 & 20.8 & 21.6 & 21.6 & 21.6 & 21.6 & 21.3 & 21.3 & 21.3 & 21.3 & 21.6 & 21.6 & 21.3 & 21.3 \\
\hline $5 \mathrm{~min}$ & 0.098 & 0.057 & 0.042 & 0.105 & 0.046 & 0.039 & 0.046 & 0.058 & 0.355 & 0.023 & 0.061 & 0.014 & 0.236 & 0.044 & 0.071 & 0.012 \\
\hline 10 & 0.101 & 0.056 & 0.042 & 0.017 & 0.04 & 0.028 & 0.046 & 0.032 & 0.079 & 0.009 & 0.079 & 0.008 & 0.092 & 0.041 & 0.058 & 0.007 \\
\hline 15 & 0.091 & 0.055 & 0.051 & 0.015 & 0.037 & 0.031 & 0.058 & 0.029 & 0.081 & 0.009 & 0.085 & 0.011 & 0.073 & 0.044 & 0.064 & 0.009 \\
\hline 20 & 0.082 & 0.052 & 0.059 & 0.013 & 0.043 & 0.037 & 0.056 & 0.031 & 0.084 & 0.011 & 0.083 & 0.014 & 0.072 & 0.049 & 0.07 & 0.01 \\
\hline 25 & 0.076 & 0.049 & 0.057 & 0.013 & 0.04 & 0.034 & 0.052 & 0.031 & 0.075 & 0.012 & 0.076 & 0.018 & 0.072 & 0.047 & 0.055 & 0.011 \\
\hline 30 & 0.072 & 0.044 & 0.056 & 0.016 & 0.039 & 0.029 & 0.052 & 0.031 & 0.071 & 0.013 & 0.062 & 0.02 & 0.07 & 0.047 & 0.05 & 0.011 \\
\hline 35 & 0.067 & 0.044 & 0.056 & 0.015 & 0.034 & 0.028 & 0.047 & 0.033 & 0.062 & 0.014 & 0.059 & 0.023 & 0.069 & 0.046 & 0.05 & 0.011 \\
\hline 40 & 0.065 & 0.04 & 0.055 & 0.017 & 0.033 & 0.024 & 0.045 & 0.032 & 0.055 & 0.015 & 0.057 & 0.025 & 0.059 & 0.045 & 0.046 & 0.012 \\
\hline 45 & 0.058 & 0.041 & 0.05 & 0.017 & 0.031 & 0.023 & 0.043 & 0.034 & 0.052 & & 0.053 & 0.027 & 0.056 & 0.041 & 0.043 & 0.014 \\
\hline 50 & 0.052 & 0.036 & 0.046 & 0.02 & 0.029 & 0.02 & 0.038 & 0.031 & 0.049 & 0.02 & 0.05 & 0.029 & 0.054 & 0.039 & 0.037 & 0.017 \\
\hline 55 & 0.048 & 0.034 & 0.045 & 0.023 & 0.028 & 0.021 & 0.037 & 0.027 & 0.044 & 0.02 & 0.047 & 0.028 & 0.05 & 0.039 & 0.038 & 0.021 \\
\hline 60 & 0.047 & 0.034 & 0.043 & 0.024 & 0.026 & 0.018 & 0.035 & 0.028 & 0.043 & 0.022 & 0.045 & 0.029 & 0.041 & 0.035 & 0.038 & 0.024 \\
\hline 70 & 0.044 & 0.031 & 0.04 & 0.021 & 0.021 & 0.016 & 0.033 & 0.026 & 0.037 & 0.021 & 0.038 & 0.025 & 0.041 & 0.034 & 0.033 & 0.021 \\
\hline 80 & 0.038 & 0.026 & 0.039 & 0.021 & 0.018 & 0.013 & 0.029 & 0.025 & 0.034 & 0.021 & 0.038 & 0.024 & 0.04 & 0.034 & 0.033 & 0.019 \\
\hline 90 & 0.033 & 0.024 & 0.034 & 0.017 & 0.019 & 0.013 & 0.025 & 0.022 & 0.031 & 0.018 & 0.036 & 0.025 & 0.036 & 0.028 & 0.027 & 0.017 \\
\hline 100 & 0.029 & & & & & & & & & & & & & & & \\
\hline 110 & 0.026 & & & & & & & & & & & & & & & \\
\hline 120 & 0.024 & & & & & & & & & & & & & & & \\
\hline $\begin{array}{c}\text { \% change } \\
B A C\end{array}$ & & $35 \%$ & & $50 \%$ & & $23 \%$ & & $27 \%$ & & $79 \%$ & & $63 \%$ & & $42 \%$ & & $70 \%$ \\
\hline $\begin{array}{l}P \text { value } \\
(\text { t test })\end{array}$ & & $<0.001$ & & 0.003 & & $<0.001$ & & 0.001 & & 0.013 & & $<0.001$ & & 0.026 & & $<0.001$ \\
\hline $\begin{array}{c}\text { Pearson's } \\
\quad R\end{array}$ & & 0.986 & & -0.296 & & 0.974 & & 0.370 & & 0.186 & & -0.828 & & 0.390 & & -0.768 \\
\hline
\end{tabular}

Table 3. Normalized blood alcohol concentration (BAC) at $20 \mathrm{gm}$ intake of alcohol (20 gm = 2 standard drinks) for sessions 1 - 16 control (C) versus active (A) RM88 for 90 minutes. Corresponding missing values were paired and not included in statistical analyses. Subject 2 undertook paired sessions 2 - 5, all other subjects only one paired session.

\begin{tabular}{ccccccccccccccccc}
\hline Session & $1 \mathrm{C}$ & $1 \mathrm{~A}$ & $\mathbf{2 C}$ & $\mathbf{2 A}$ & $\mathbf{3 C}$ & $\mathbf{3 A}$ & $\mathbf{4 C}$ & $\mathbf{4 A}$ & $\mathbf{5 C}$ & $\mathbf{5 A}$ & $\mathbf{6 C}$ & $\mathbf{6 A}$ & $\mathbf{7 C}$ & $\mathbf{7 A}$ & $\mathbf{8 C}$ & $\mathbf{8 A}$ \\
\hline $5 \mathrm{~min}$ & 0.232 & 0.030 & 0.276 & 0.071 & 0.136 & 0.079 & 0.084 & 0.086 & 0.075 & 0.042 & 0.030 & 0.016 & 0.050 & 0.044 & 0.333 & 0.028 \\
10 & 0.086 & 0.017 & 0.210 & 0.030 & 0.051 & 0.054 & 0.053 & 0.022 & 0.050 & 0.029 & 0.032 & 0.017 & 0.036 & 0.153 & 0.046 & 0.027 \\
15 & 0.045 & 0.014 & 0.177 & 0.032 & 0.040 & 0.033 & 0.048 & 0.015 & 0.031 & 0.024 & 0.034 & 0.015 & 0.040 & 0.051 & 0.047 & 0.028 \\
20 & 0.036 & 0.014 & 0.143 & 0.029 & 0.038 & 0.038 & 0.039 & 0.015 & 0.033 & 0.024 & 0.033 & 0.018 & 0.038 & 0.040 & 0.046 & 0.027 \\
25 & 0.034 & 0.013 & 0.119 & 0.029 & 0.037 & 0.029 & 0.034 & 0.016 & 0.033 & 0.019 & 0.033 & 0.022 & 0.040 & 0.040 & 0.041 & 0.025 \\
30 & 0.033 & 0.011 & 0.101 & 0.028 & 0.034 & 0.040 & 0.029 & 0.015 & 0.033 & 0.018 & 0.033 & 0.022 & 0.038 & 0.039 & 0.040 & 0.024 \\
\hline
\end{tabular}




\section{Continued}

\begin{tabular}{|c|c|c|c|c|c|c|c|c|c|c|c|c|c|c|c|c|}
\hline 35 & 0.029 & 0.013 & 0.084 & 0.023 & 0.031 & 0.025 & 0.026 & 0.012 & 0.030 & 0.016 & 0.032 & 0.023 & & & 0.039 & 0.023 \\
\hline 40 & 0.029 & 0.019 & 0.074 & 0.024 & 0.032 & 0.024 & 0.020 & 0.014 & 0.028 & & 0.030 & 0.024 & 0.033 & 0.038 & 0.037 & 0.023 \\
\hline 50 & 0.023 & 0.016 & 0.061 & 0.018 & 0.028 & 0.021 & 0.015 & 0.010 & 0.024 & 0.016 & 0.026 & 0.019 & 0.030 & 0.031 & 0.036 & 0.019 \\
\hline 55 & 0.027 & 0.018 & 0.054 & 0.017 & & 0.000 & 0.008 & 0.008 & 0.022 & & 0.028 & 0.016 & & & 0.032 & 0.018 \\
\hline 60 & 0.027 & 0.016 & 0.049 & 0.015 & 0.023 & 0.017 & 0.000 & 0.008 & 0.020 & 0.015 & 0.026 & 0.017 & 0.028 & 0.028 & 0.031 & 0.017 \\
\hline 70 & 0.023 & 0.014 & 0.043 & 0.009 & 0.021 & 0.016 & & 0.007 & 0.021 & 0.014 & 0.021 & 0.014 & 0.022 & 0.029 & 0.029 & 0.014 \\
\hline 80 & 0.016 & 0.011 & 0.030 & 0.008 & 0.016 & 0.015 & & 0.000 & 0.017 & & 0.021 & 0.013 & & & 0.027 & 0.013 \\
\hline 90 & 0.013 & 0.008 & 0.024 & 0.007 & 0.016 & 0.011 & & & 0.014 & 0.016 & 0.017 & 0.011 & 0.020 & 0.022 & 0.022 & 0.011 \\
\hline $5 \mathrm{~min}$ & 0.082 & 0.047 & 0.040 & 0.101 & 0.043 & 0.036 & 0.043 & 0.054 & 0.333 & 0.022 & 0.057 & 0.013 & 0.219 & 0.041 & 0.067 & 0.011 \\
\hline 10 & 0.084 & 0.047 & 0.040 & 0.016 & 0.037 & 0.026 & 0.043 & 0.030 & 0.074 & 0.008 & 0.074 & 0.008 & 0.085 & 0.038 & 0.054 & 0.007 \\
\hline 15 & 0.076 & 0.046 & 0.049 & 0.014 & 0.034 & 0.029 & 0.054 & 0.027 & 0.076 & 0.008 & 0.080 & 0.010 & 0.068 & 0.041 & 0.060 & 0.008 \\
\hline 20 & 0.068 & 0.043 & 0.057 & 0.013 & 0.040 & 0.034 & 0.052 & 0.029 & 0.079 & 0.010 & 0.078 & 0.013 & 0.067 & 0.046 & 0.066 & 0.009 \\
\hline 25 & 0.063 & 0.041 & 0.055 & 0.013 & 0.037 & 0.031 & 0.048 & 0.029 & 0.070 & 0.011 & 0.071 & 0.017 & 0.067 & 0.044 & 0.052 & 0.010 \\
\hline 30 & 0.060 & 0.037 & 0.054 & 0.015 & 0.036 & 0.027 & 0.048 & 0.029 & 0.067 & 0.012 & 0.058 & 0.019 & 0.065 & 0.044 & 0.047 & 0.010 \\
\hline 35 & 0.056 & 0.037 & 0.054 & 0.014 & 0.031 & 0.026 & 0.044 & 0.031 & 0.058 & 0.013 & 0.055 & 0.022 & 0.064 & 0.043 & 0.047 & 0.010 \\
\hline 40 & 0.054 & 0.033 & 0.053 & 0.016 & 0.031 & 0.022 & 0.042 & 0.030 & 0.052 & 0.014 & 0.054 & 0.023 & 0.055 & 0.042 & 0.043 & 0.011 \\
\hline 45 & 0.048 & 0.034 & 0.048 & 0.016 & 0.029 & 0.021 & 0.040 & 0.031 & 0.049 & & 0.050 & 0.025 & 0.052 & 0.038 & 0.040 & 0.013 \\
\hline 55 & 0.040 & 0.028 & 0.043 & 0.022 & 0.026 & 0.019 & 0.034 & 0.025 & 0.041 & 0.019 & 0.044 & 0.026 & 0.046 & 0.037 & 0.036 & 0.020 \\
\hline 60 & 0.039 & 0.028 & 0.041 & 0.023 & 0.024 & 0.017 & 0.032 & 0.026 & 0.040 & 0.021 & 0.042 & 0.027 & 0.038 & 0.033 & 0.036 & 0.023 \\
\hline 70 & 0.037 & 0.026 & 0.038 & 0.020 & 0.019 & 0.015 & 0.031 & 0.024 & 0.035 & 0.020 & 0.036 & 0.023 & 0.038 & 0.032 & 0.031 & 0.020 \\
\hline 80 & 0.032 & 0.022 & 0.038 & 0.020 & 0.017 & 0.012 & 0.027 & 0.023 & 0.032 & 0.020 & 0.036 & 0.023 & 0.037 & 0.032 & 0.031 & 0.018 \\
\hline 90 & 0.027 & 0.020 & 0.033 & 0.016 & 0.018 & 0.012 & 0.023 & 0.020 & 0.029 & 0.017 & 0.034 & 0.023 & 0.033 & 0.026 & 0.025 & 0.016 \\
\hline
\end{tabular}

In the control sessions seven subjects reported feeling slightly to moderately drunk. One subject that was moderately drunk on control reported being only slightly drunk on the active compound session. Three female subjects from one family reported facial flushing from the active session. This was thought to be attributed to niacinamide in the active combination as there was a family history of histamine release to this compound. There were no other side effects reported or observed.

\section{Discussion}

Results of this study provide evidence that herbal and natural compounds have a positive effect on alcohol clearance kinetics. The study design was simple and participants (moderate social drinkers) were interested to know their respective rates of alcohol metabolism. Further study is required to evaluate any therapeu- 
tic benefit to high risk alcoholic groups. The highly significant reduction in BAC in the 5 to 30 minute testing period shows RM88 was able to reduce the maximum peak of alcohol, potentially avoiding loss of control in drinking behaviour. The socio-economic cost from the side effects of alcohol are substantial in many countries causing increased domestic violence, increased deaths from motor vehicles and guns, and loss of work (Table 4). Limitations of this open label paired session study were subject recruitment was restricted to social drinkers with a history of moderate alcohol intake, there was no testing of binge drinkers or chronic alcoholics. There was also a lack of a cookie placebo in this study, however there is no evidence that a placebo can negate an objective breathalyzer test.

The major pathway of alcohol metabolism is via the liver enzyme ADH. One subject using prednisone and hydroxychloroquine had a significantly increased BAC but the pharmacokinetic mechanism(s) cannot be established from this study. A review of the literature showed prednisone is converted to the pharmacologically active prednisolone in the liver by another dehydrogenase enzyme [17] but there has been no report of direct $\mathrm{ADH}$-prednisone or $\mathrm{ADH}$-hydroxychloroquine interaction to upregulate the $\mathrm{BAC}$ as observed in this study.

Herbal extracts and other natural compounds are widely used by the public particularly for conditions such as persistent pain [18]. The use of comprehensive herbal formulas to act synergistically has been studied and developed for thousands of years in China and other countries. The investigator's approach was to adopt this method applying knowledge from an international herbal

Table 4. Comparison of blood alcohol concentration means (SD) of normalized data for 20 gm alcohol consumption, control versus active RM88 ( $p=0.00026)$.

\begin{tabular}{ccc}
\hline Time (min) & Control BAC mean $(\mathrm{SD})$ & RM88 BAC mean $(\mathrm{SD})$ \\
\hline 5 & $0.131(0.093)$ & $0.045(0.021)$ \\
10 & $0.066(0.027)$ & $0.033(0.02)$ \\
15 & $0.060(0.022)$ & $0.025(0.011)$ \\
20 & $0.057(0.02)$ & $0.025(0.011)$ \\
25 & $0.052(0.017)$ & $0.024(0.009)$ \\
30 & $0.048(0.014)$ & $0.024(0.009)$ \\
35 & $0.045(0.013)$ & $0.022(0.007)$ \\
40 & $0.042(0.012)$ & $0.024(0.006)$ \\
45 & $0.039(0.011)$ & $0.023(0.006)$ \\
50 & $0.036(0.01)$ & $0.021(0.006)$ \\
55 & $0.034(0.009)$ & $0.020(0.006)$ \\
60 & $0.031(0.009)$ & $0.021(0.006)$ \\
70 & $0.03(0.007)$ & $0.018(0.006)$ \\
80 & $0.027(0.007)$ & $0.016(0.006)$ \\
90 & $0.023(0.006)$ & $0.016(0.005)$ \\
\hline & & \\
\hline
\end{tabular}


pharmacopeia perspective. Most well studied herbal products and natural compounds of potential ADH activity that were identified in the preliminary assessment were available for the RM88 formulation. One key advantage of using natural compounds is low cost availability and sufficient documented research pertaining to safety. There were other natural compounds considered but these were from rare herbs. In addition, marine and insect peptides were considered but were cost prohibitive to produce for RM88. The tested formula was a powder mix that can be dissolved in a drink or added to a cookie recipe and suitable for use by the general population. Adding appropriate preservatives would provide for a commercial product with adequate shelf life for retail purposes. The use of the cookie method augments normal social drinking habits of consuming food while drinking alcohol. Importantly, for the delivery method with RM88 there was no need for medical interventions such as local injections or intravenous administration. The cookies were consumed 1 hour prior to alcohol to enable absorption of the herbs. Late in the study three additional sessions with RM88 consumed only 20 minutes prior to alcohol yielded improved BAC kinetics (data not presented). Further work is needed on optimizing this variable.

The analysis of the data points over 5 to 90 minutes showed a rapid reduction of alcohol in the first 15 minutes, subsequently followed by a constant rate of metabolism in the control and RM88 sessions. Spurious data can be recorded in this initial 15 minute period as local absorption of alcohol can still be present in oral mucosal tissue, potentially resulting in higher than expected BAC. Kinetic order profiles were not conducted in this study as the rate of consumption of alcohol was not strictly controlled. Pearson correlation of paired BAC measurements was strong $(>0.7)$ in $63 \%$ (10/16) of paired sessions.

The reduced BAC is a positive effect for improving outcomes from a regenerative medicine perspective. Stem cells are a key component for health and longevity. Extracellular matrix cues by cytokines, growth factors and peptides can greatly affect stem cell expansion and differentiation. In vitro evidence of polyphenols from herbal products has shown beneficial effects for stem cells, while toxins and high concentrations of substances such as alcohol are detrimental [9].

\section{Conclusions}

This exploratory study investigated the possibility that elements of traditional and folklore medicine could have applications for alcohol reduction strategies. Results showed that this is possible, and with further research to develop specific formulas for the prevention of binge drinking and therapeutic interventions to treat alcoholism. In the developing area of regenerative medicine, a reduced BAC would likely improve patient outcomes from stem cell therapy and lessen damaging behaviours of addiction [19] [20]. Table 5 identifies the negative effects from alcohol overuse triggering social misbehaviour, crime and hospitalizations that can be frequent in society. To date there has been no successful synthetic drug developed to decrease alcohol. 
Table 5. Summary of the prevalence, medical consequences and economic burden of alcohol consumption in 4 countries.

\begin{tabular}{|c|c|c|c|}
\hline Country & Prevalence and social effects & Medical consequences & Economic burden \\
\hline $\begin{array}{c}\text { USA } \\
{[21]-[25]}\end{array}$ & $\begin{array}{l}12.7 \% \text { of adults meet the criteria } \\
\text { for alcohol use disorder (AUD) } \\
10 \% \text { of U.S. children live with a parent } \\
\text { with alcohol problems ( } 2012 \text { ) } \\
10,500 \text { motor vehicle deaths (2016) } \\
111 \text { million self-reported episodes } \\
\text { of alcohol-impaired driving among } \\
\text { U.S. adults each year }\end{array}$ & $\begin{array}{l}88,000 \text { deaths annually } \\
\text { (cirrhosis, cancer) } \\
\text { Alcohol involved in } 50 \% \text { of } \\
\text { homicides and } 33 \% \text { of suicides }\end{array}$ & $\begin{array}{l}\text { USD } 249 \text { billion (2010) } \\
\text { Early mortality due to alcohol } \\
\text { ( } \$ 75 \text { billion of the total), lost } \\
\text { productivity and absenteeism at work } \\
\text { ( } \$ 82 \text { billion), health-care costs } \\
\text { ( } \$ 28 \text { billion), crime ( } \$ 25 \\
\text { billion) and car crashes ( } \$ 13 \text { billion) }\end{array}$ \\
\hline $\begin{array}{l}\text { United Kingdom } \\
\qquad[26]\end{array}$ & $\begin{array}{l}2 \%-3 \% \text { of population are dependent } \\
\text { drinkers }\end{array}$ & $\begin{array}{l}1.17 \text { million hospital admissions } \\
\text { due to alcohol (2017) }\end{array}$ & 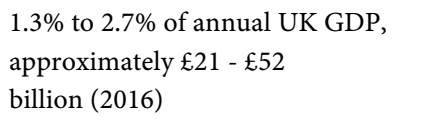 \\
\hline PR China [27] & Alcohol use disorder $9.3 \%$ of males & 310,000 deaths annually (2010) & \\
\hline $\begin{array}{c}\text { Australia } \\
\text { [28] [29] [30] }\end{array}$ & $\begin{array}{l}6 \% \text { of population drink alcohol daily, } 18 \% \\
\text { drink most days/week, } 20 \% \text { of employed } \\
\text { people are high risk drinkers, } 22 \% \\
\text { experienced abuse of fear related to alcohol }\end{array}$ & $\begin{array}{l}4186 \text { deaths (2017) annually } \\
144,000 \text { hospital admissions (2013) }\end{array}$ & AUD $\$ 14.4$ billion (2010) \\
\hline
\end{tabular}

Results of the normalized data showed that the herbal formula was effective in reducing $\mathrm{BAC}$ on all data points to less than $0.05 \%$, a threshold of legality in many countries for the public to drive a motor vehicle. This occurred at the 5 minute interval and thereafter, demonstrating the efficacy of the formula in rapidly reducing $\mathrm{BAC}$ to an acceptable legal level. In comparison, it took 30 minutes for the control session to reach less than $0.05 \% \mathrm{BAC}$.

The cost to society of alcohol abuse is estimated to be approximately $1 \%$ of world gross domestic product (world GDP US $\$ 89$ trillion 2019). The developed formula could provide an active prevention and treatment strategy to governments, health authorities and hospitals, companies engaged in alcohol manufacturing, and liquor sales outlets. In addition, there is the opportunity to reduce personal and family tragedy from alcohol related road deaths and injuries, and domestic violence. This may be particularly pertinent where increased consumption of alcoholic beverages has caused a reported consequential increase in domestic violence. Lockdowns from the current coronavirus trigger fear, anxiety and psychological stress. This issue, coupled with loss of employment from industry shutdown, and the prospect of re-emergence of coronavirus mutations suggests stress and alcohol will likely be present throughout the world for an extended period.

\section{Conflicts of Interest}

The author declares information from this study may be used for future commercial purposes.

\section{References}

[1] https://www.alcohol.org/guides/global-drinking-demographics

[2] World Health Organization (2011) Global Status Report on Alcohol and Health. 
https://www.who.int/substance abuse/publications/global alcohol report/msbgsru profiles.pdf

[3] Thavorncharoensap, M., Teerawattananon, Y., Yothasamut, J., Lertpitakpong, C. and Chaikledkaew, U. (2009) The Economic Impact of Alcohol Consumption: A Systematic Review. Substance Abuse Treatment, Prevention, and Policy, 4, Article 20. https://doi.org/10.1186/1747-597X-4-20

[4] Martinez-Hurtado, J.L., Calo-Fernandez, B. and Vazquez-Padin, J. (2018) Preventing and Mitigating Alcohol Toxicity: A Review on Protective Substances. Beverages, 4, 39. https://doi.org/10.3390/beverages4020039

[5] Crowley, P. (2015) Long-Term Drug Treatment of Patients with Alcohol Dependence. Australian Prescriber, 38, 41-43. https://doi.org/10.18773/austprescr.2015.015

[6] World Health Organization (2018). https://www.who.int/news-room/fact-sheets/detail/alcohol

[7] Santillano, D.R., Kumar, L.S., Prock, T.L., Camarillo, C., Tingling, J.D. and Miranda, R.C. (2005) Ethanol Induces Cell-Cycle Activity and Reduces Stem Cell Diversity to Alter Both Regenerative Capacity and Differentiation Potential of Cerebral Cortical Neuroepithelial Precursors. BMC Neuroscience, 13, Article No. 59. https://doi.org/10.1186/1471-2202-6-59

[8] Di Rocco, G., Baldari, S., Pani, G. and Toietta, G. (2019) Stem Cells under the Influence of Alcohol: Effects of Ethanol Consumption on Stem/Progenitor Cells. Cellular and Molecular Life Sciences, 76, 231-244. https://doi.org/10.1007/s00018-018-2931-8

[9] Serio, R.N. and Gudas, L.J. (2020) Modification of Stem Cell States by Alcohol and Acetaldehyde. Chemico-Biological Interactions, 316, Article ID: 108919. https://doi.org/10.1016/j.cbi.2019.108919

[10] Jiang, Y., Zhang, T., Kusumanchi, P., Han, S., Yang, Z. and Liangpunsakul, S. (2020) Alcohol Metabolizing Enzymes, Microsomal Ethanol Oxidizing System, Cytochrome P450 2E1, Catalase, and Aldehyde Dehydrogenase in Alcohol-Associated Liver Disease. Biomedicines, 8, 50. https://doi.org/10.3390/biomedicines8030050

[11] Lee, F.C., Ko, J.H., Park, J.K. and Lee, J.S. (1987) Effects of Panax Ginseng on Blood Alcohol Clearance in Man. Clinical and Experimental Pharmacology and Physiology, 14, 543-546. https://doi.org/10.1111/j.1440-1681.1987.tb01510.x

[12] Lee, M.H., Kwak, J.H., Jeon, G., Lee, J.W., Seo, J.H., Lee, H.S. and Lee, J.H. (2014) Red Ginseng Relieves the Effects of Alcohol Consumption and Hangover Symptoms in Healthy Men: A Randomized Crossover Study. Food \& Function, 5, 528-534. https://doi.org/10.1039/c3fo60481k

[13] Siegel, R.K. (1979) Ginseng Abuse Syndrome. Problems with the Panacea. Journal of the American Medical Association, 241, 1614-1615. https://doi.org/10.1001/jama.1979.03290410046024

[14] Kiefer, D. and Pantuso, T. (2003) Panax Ginseng. American Family Physician, 68, 1539-1542.

[15] Xie, C.I., Lin, R.C., Antony, V., Lumeng, L., Li, T.K., Mai, K., et al. (1994) Daidzin, an Antioxidant Isoflavonoid, Decreases Blood Alcohol Levels and Shortens Sleep Time Induced by Ethanol Intoxication. Alcoholism: Clinical and Experimental Research, 18, 1443-1447. https://doi.org/10.1111/j.1530-0277.1994.tb01448.x

[16] Rezvani, A.H., Overstreet, D.H. and Leef, Y.W. (1995) Attenuation of Alcohol Intake by Ibogaine in Three Strains of Alcohol-Preferring Rats. Pharmacology Biochemistry and Behavior, 52, 615-620. https://doi.org/10.1016/0091-3057(95)00152-M 
[17] Cooper, M.S., Blumsohn, A., Goddard, P.E., Bartlett, W.A., Shackleton, C.H., Eastell, et al. (2003) 11 $\beta$-Hydroxysteroid Dehydrogenase Type 1 Activity Predicts the Effects of Glucocorticoids on Bone. The Journal of Clinical Endocrinology \& Metabolism, 88, 3874-3877. https://doi.org/10.1210/jc.2003-022025

[18] Vickers, E.R. (2020) Natural Compounds for Treating Pain. Pain Management Today (Royal Australian College of General Practitioners), 6, 72-75.

https://painmanagementtoday.com.au/2019/july/regular-series/natural-compounds -treating-pain

[19] Shparberg, R., Braunsteiner, N. and Vickers, E.R. (2018) Laboratory Training Guidelines for Clinicians Undertaking Stem Cell Therapy. Journal of Stem Cell Research and Therapy, 8, 1-10.

https://www.longdom.org/open-access/laboratory-training-guidelines-for-clinicians -undertaking-stem-celltherapy-2157-7633-1000427.pdf

[20] Braunsteiner, N.E., Vickers, E.R. and Shparberg, R. (2018) Psychological Issues for Patients Undergoing Stem Cell Therapy and Regenerative Medicine. Open Journal of Regenerative Medicine, 7, 1-17. https://doi.org/10.4236/ojrm.2018.71001

[21] https://www.niaaa.nih.gov/publications/brochures-and-fact-sheets/alcohol-facts-an d-statistics

[22] https://www.healthline.com/health/facts-about-alcohol\#facts

[23] https://www.cdc.gov/motorvehiclesafety/impaired driving/impaired-drv factsheet. $\underline{\mathrm{html}}$

[24] https://gunsandamerica.org/story/19/12/17/relationship-between-alcohol-gun-viole nce

[25] https://www.washingtonpost.com/news/wonk/wp/2015/10/16/the-hidden-cost-of-y our-drinking-habit

[26] https://www.drinkaware.co.uk/research/data/consequences

[27] https://www.thelancet.com/journals/langlo/article/PIIS2214-109X(15)70017-3/fullte $\underline{\mathrm{xt}}$

[28] https://www.aihw.gov.au/reports/alcohol/alcohol-tobacco-other-drugs-australia/co ntents/impacts/economic-impacts

[29] https://nadk.flinders.edu.au/kb/alcohol/consumption-patterns/frequency-consumpt ion/how-often-do-australians-drink-alcohol

[30] https://www.abs.gov.au/ausstats/abs@.nsf/Lookup/by\%20Subject/3303.0 2017 Mai n\%20Features Deaths\%20due\%20to\%20harmful\%20alcohol\%20consumption $\% 20 \mathrm{i}$ n\%20Australia 4 